\title{
A Prospective Pilot Study of Single 19 Gy Fraction High-Dose-Rate Brachytherapy for Favorable-Risk Adenocarcinoma of the Prostate
}

Scott Dahlbeck ${ }^{1}$, Chase C. Hansen ${ }^{1 *}$, Werner deRiese ${ }^{3}$, Robert Kagan $A^{4}$, Carlos Torres ${ }^{1}$, Maurizio Chiriva-Internati $D^{2}$, Everardo Cobos ${ }^{2}$, Jose A. Figueroa ${ }^{2}$, Diane Nguyen ${ }^{2}$, Lukman Tijani ${ }^{2}$ and Jaden D. Evans ${ }^{1,5}$

${ }^{1}$ Departments of Radiation Oncology, Texas Tech University Health Sciences Center School of Medicine, Lubbock, Texas, USA

${ }^{2}$ Departments of Hematology and Oncology, Texas Tech University Health Sciences Center School of Medicine, Lubbock, Texas, USA

${ }^{3}$ Departments of Urology, Texas Tech University Health Sciences Center School of Medicine, Lubbock, Texas, USA

${ }^{4}$ Department of Radiation Oncology, Southern California Permanente Medical Group, Los Angeles, California, USA

${ }^{5}$ Department of Internal Medicine, Intermountain Medical Center, Murray, Utah, USA

*Corresponding author: Chase C. Hansen, Department of Radiation Oncology, Texas Tech University Health Sciences Center, Lubbock, TX -79415, USA, Tel: (435) 215-3574; E-mail: chase.hansen@ttuhsc.edu

Received date: Jan 21, 2015, Accepted date: Mar 05, 2015, Publication date: Mar 09, 2015

Copyright: $\odot 2015$ Dahlbeck S, et al. This is an open-access article distributed under the terms of the Creative Commons Attribution License, which permits unrestricted use, distribution, and reproduction in any medium, provided the original author and source are credited.

\begin{abstract}
Objective: To evaluate the acute genitourinary (GU) and gastrointestinal (GI) toxicities, health-related quality of life (HRQOL) factors, biochemical control rates, and technical feasibility of high-dose-rate (HDR) brachytherapy as monotherapy for prostate cancer delivered in a single fraction.

Methods: A single-institution, prospective pilot study evaluating 6 patients with low- and intermediate-risk prostate cancer treated in 2013. Patients received a single 19 Gy fraction as HDR monotherapy. Patients were assessed according to the Common Terminology Criteria for Adverse Events version 4.0, the International Index of Erectile Function (IIEF-5), the International Prostate Symptom Score (IPSS), the Expanded Prostate Cancer Index Composite-Bowel Assessment (EPIC-Bowel), a Quality of Life (QOL) Assessment, and an institutionally designed quality of care (QOC) questionnaire. Biochemical failure was defined as a prostate-specific antigen (PSA) nadir plus $2 \mathrm{ng} / \mathrm{ml}$.
\end{abstract}

Results: Patients tolerated the implant well and were all discharged home the same day by approximately 4 pm. Median follow-up was 9 months. No grade 3, 4 or 5 toxicities were observed. Two of the 6 patients (33\%) experienced grade $2 \mathrm{GU}$ toxicity. One patient (17\%) experienced grade $2 \mathrm{Gl}$ toxicity. HRQOL bowel and urinary assessments revealed a majority of complaints at 3 months, which returned to baseline at 6 months.

Conclusion: HDR brachytherapy as monotherapy for favorable-risk prostate cancer using one implant delivered in a single 19 Gy dose has acceptable acute toxicities and HRQOL reports similar to alternative treatment options.

Keywords: Acute toxicity; High-dose-rate; HDR brachytherapy; Prostate cancer; Quality of life

\section{Introduction}

Adenocarcinoma of the prostate has multiple effective treatment options for low- and intermediate-risk disease including radical prostatectomy, external beam radiotherapy (EBRT), low dose rate (LDR) or high dose rate (HDR) brachytherapy, or active surveillance. Given that no specific treatment has been shown to be significantly superior over another [1], the treatment choice is often influenced by physician bias, patient preference, and cost. Regarding radiotherapy, one option of increasing interest is that of high-dose-rate (HDR) brachytherapy with iridium-192. In recent years, there has been a trend to further develop HDR brachytherapy techniques and implement its use for the treatment of prostate cancer because of its excellent clinical outcomes and radiobiologic and dosimetric advantages [2]. HDR brachytherapy has many attractive advantages over low-dose-rate (LDR) brachytherapy. These advantages include no radiation exposure to family or staff, pre-treatment delivery of an optimized dose plan, and a more favorable side effect profile [3-5]. The major HDR advantages over external beam radiotherapy include timerelated convenience and cost benefits to the patient. Furthermore, given the growing body of knowledge concerning the radiobiology of prostate cancer suggestive of a low alpha-beta ratio, hypofractionated schedules have gained considerable interest amongst investigators [6-8].

Prada et al. recently published the first report of HDR brachytherapy as monotherapy in one fraction for patients with lowand intermediate-risk prostate cancer [9]. Patients were treated with $19 \mathrm{~Gy}$ in a single fraction and excellent biochemical control rates were reported. Additionally, Prada et al. performed transperineal hyaluronic acid injections into the perirectal fat to minimize rectal toxicity. This single fraction HDR brachytherapy was well tolerated by all patients with minimal to no genitourinary or gastrointestinal toxicity.

Given the promising results of Prada's report, a prospective pilot study was initiated to evaluate single-fraction HDR brachytherapy used as monotherapy for favorable-risk prostate cancer. The purpose 
Citation: Dahlbeck S, Hansen CC, deRiese W, Kagan AR, Torres C, et al. (2015) A Prospective Pilot Study of Single 19 Gy Fraction High-DoseRate Brachytherapy for Favorable-Risk Adenocarcinoma of the Prostate. J Nucl Med Radiat Ther 6: 215. doi: $10.4172 / 2155-9619.1000215$

Page 2 of 7

of which was to determine the technical feasibility, toxicity, and efficacy of this single-fraction approach without the use of the transperineal hyaluronic acid injections. The present article is a report of technical feasibility, acute toxicity, patient satisfaction, and biochemical control rates.

\section{Materials and Methods}

\section{Study design}

We conducted a single-institution prospective pilot study after approval of the appropriate internal review board. A total of 6 male patients were enrolled and treated with high-dose-rate brachytherapy as monotherapy for low- and intermediate-risk adenocarcinoma of the prostate. Treatment was given as a single implant delivered in one 19 Gy fraction. Patient disease was staged using the 7th edition AJCC staging manual and stratified into risk categories of low, intermediate, and high risk according to the version 2.2012 National Cancer Cooperative Network (NCCN) risk factors.

\section{Selection criteria}

Patient accrual was accomplished by informing referring physicians in the community of the study by letter. Patients felt to be eligible for enrollment on the study were referred to radiation oncology for a prestudy evaluation to determine eligibility. Please see Table 1 for inclusion and exclusion criteria.

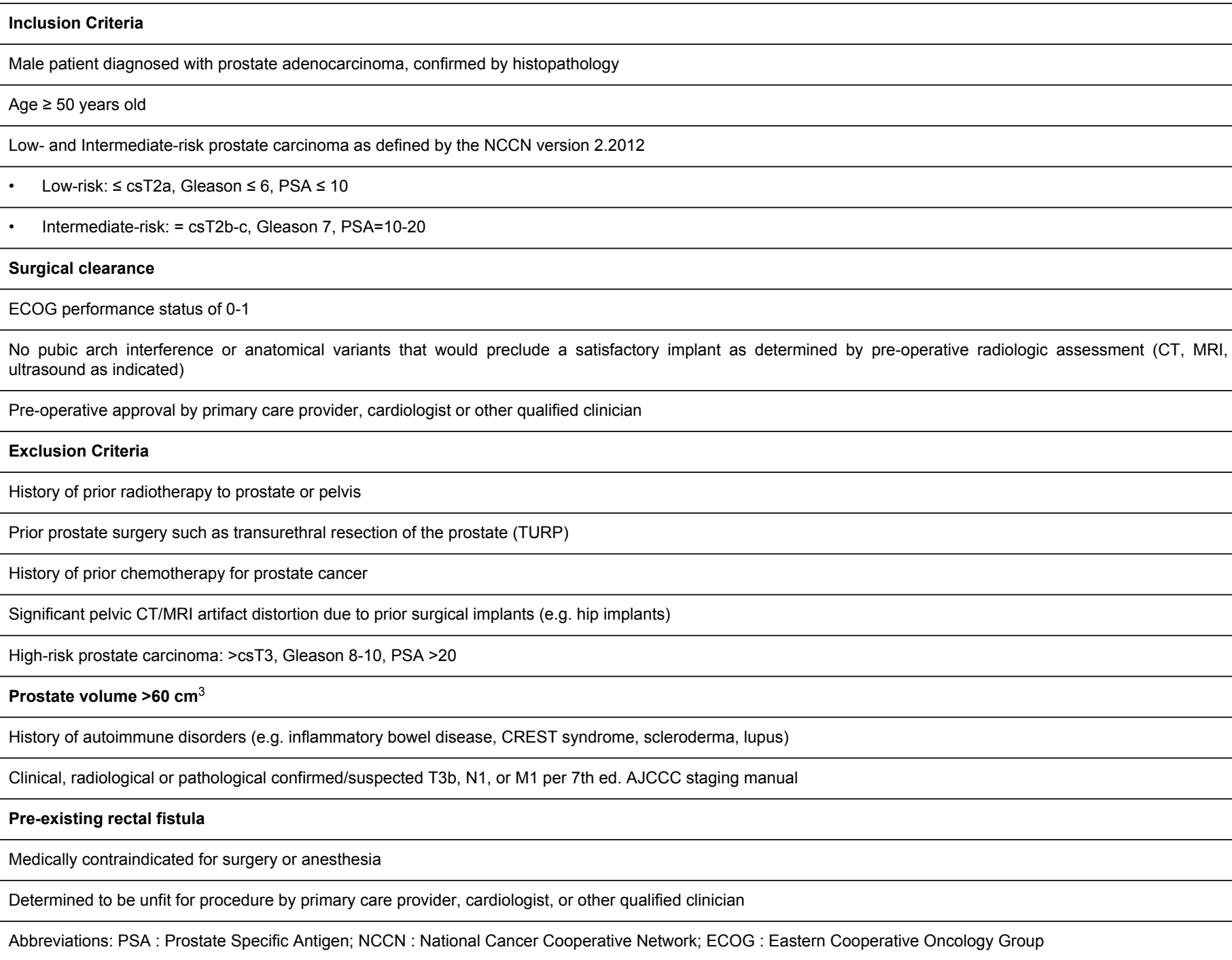

Table 1: Patient selection criteria.

\section{Pre-brachytherapy evaluation}

Once enrolled on the protocol, each patient was cleared surgically by his primary care provider or cardiologist and evaluated in the preop anesthesia clinic to obtain anesthesia clearance. Complete history and physical examination findings including a digital rectal exam were documented for all patients. The pre-op evaluation included routine labs, ECG, and a chest X-ray. For additional information, a CT or MRI of the pelvis was done to rule out potential pubic arch interference. Baseline serum PSA levels were drawn within one month prior to the 
Citation: Dahlbeck S, Hansen CC, deRiese W, Kagan AR, Torres C, et al. (2015) A Prospective Pilot Study of Single 19 Gy Fraction High-DoseRate Brachytherapy for Favorable-Risk Adenocarcinoma of the Prostate. J Nucl Med Radiat Ther 6: 215. doi: $10.4172 / 2155-9619.1000215$

Page 3 of 7

procedure. Baseline patient questionnaires were completed by each patient that included the International Index of Erectile Function (IIEF-5), the International Prostate Symptom Score (IPSS), the Expanded Prostate Cancer Index Composite - Bowel Assessment (EPIC-Bowel), a Quality of Life (QOL) Assessment, and an institutionally designed quality of care (QOC) questionnaire. The perceived quality of life (QOL) assessment was a one page scale marked at 0.5 intervals from $0=$ worst possible state of health to $100=$ best possible state of health, and the patient drew a line at the level of their perceived overall quality of life. The quality of care (QOC) questionnaire included 5 levels of care: $1=$ very dissatisfied with care plan, 2=dissatisfied with care plan, $3=$ neutral (no impact) regarding care, $4=$ satisfied with care plan, $5=$ very satisfied with care plan. Pre-op instructions included a prescription for a polyethylene glycol electrolyte solution to be taken the evening prior to the procedure, clear liquid diet for 24 hours, NPO after midnight, and a sodium phosphate rectal enema to be administered the morning of the procedure.

\section{HDR brachytherapy}

Patients were given IV antibiotics, typically gentamicin and metronidazole, just prior to the procedure. Compression stockings were used for lower extremity venous thromboembolism prophylaxis. Dissimilar to the Prada method [9], hyaluronic acid injection into the posterior prostatic space was not utilized. Prada et al. used this method to displace the rectum posteriorly away from the prostate in an attempt to protect the rectum. This technique is not FDA approved in the United States and may not be necessary for rectal sparing. No intentional positioning to displace bowel superiorly was done during simulation and delivery of dose due to targeted DVH constraints being met without such interventions. After general and/or epidural anesthesia, the patient was placed in the dorsal lithotomy position in stirrups, cleaned and prepped in the usual sterile fashion, and a 3-way Foley catheter placed into the bladder. ProGuide needles (Nucletron, Veenendal, The Netherlands) were placed under transrectal ultrasound (TRUS) guidance in a pattern designed to cover the entire gland with an emphasis on bilateral peripheral lobe coverage. Once adequate coverage of the prostate was established with adequate sparing of $>0.5 \mathrm{~cm}$ from the rectum and $>1.5 \mathrm{~cm}$ from the urethra documented on ultrasound, the Mick-nuclear template was sutured into place on the perineal skin surface with four corner template silk sutures. Digital rectal exam was performed to confirm that no needles were within the rectum. The patient was then transferred to the Slessinger board (Slessinger Enterprises LLC, Indianapolis, IN) and placed on the hospital gurney. Once positioned on the Slessinger board, the TRUS was repeated to confirm depth of the catheters with the catheters adjusted appropriately as needed. Catheters were positioned at the bladder-prostate interface avoiding bladder wall puncture. The patient was then transferred to the CT simulation suite in the cancer center. Prior to the first CT scan, $25 \mathrm{ml}$ of contrast was placed within the bladder. The first CT scan was limited to the distal catheters to confirm correct depth positioning at the prostate-bladder interface. As needed, catheters were advanced cranially or withdrawn caudally as needed. Once ideal positioning was obtained the template was tightened to prevent catheter movement. The catheters were marked at the catheter-template interface to confirm that no movement of the catheter had occurred away from the template prior to the treatment. CT images were then obtained through the entire bladder down to the outer edge of the template. Images were transferred to an Oncentra planning station (Nucletron NV, The Netherlands) for contouring and isodose plan development. The treatment plan was typically completed within 2 hours of the simulation during which the patient remained in the same position on the Slessinger board with legs held in position with velco straps to limit lower extremity movement and to provide patient support. Please see Table 2 for treatment parameters and Table 3 for patient dose-volume-histogram constraints. HDR brachytherapy was given in the designated HDR suite under camera surveillance using an iridium-192 stepping source unit (MicroSelectron, Nucletron NV, The Netherlands). At the conclusion of the treatment, the template and catheters were pulled out in one movement. Once the patient urinated, the patient was discharged home at approximately 4 pm the same day. The patient was typically discharged on tamsulosin, pain medications, and antibiotics for 7 days.

\begin{tabular}{|l|l|l|l|}
\hline Prostate & Urethra & Rectum & Bladder \\
\hline V100 $>90 \%$ & V100<95\% & V75<1\% & V85<1\% \\
\hline V130<60\% & V125<1\% & & \\
\hline V160<30\% & & & \\
\hline Abbreviations: V100=Percentage of the structure volume receiving 100\% of the prescription dose (19 Gy). & \\
\hline
\end{tabular}

Table 2: Treatment parameters.

\begin{tabular}{|c|c|c|c|c|c|c|}
\hline & Patient 001 & Patient 002 & Patient 003 & Patient 004 & Patient 005 & Patient 006 \\
\hline \multicolumn{7}{|c|}{ Urethra } \\
\hline V100 & $67.31 \%$ & $63.55 \%$ & $60.48 \%$ & $50.36 \%$ & $36.34 \%$ & $60.56 \%$ \\
\hline V125 & $0.03 \%$ & $0.08 \%$ & $0.05 \%$ & $0 \%$ & $0 \%$ & $0 \%$ \\
\hline \multicolumn{7}{|c|}{ Bladder } \\
\hline V85 & $0 \%$ & $0.80 \%$ & $0.14 \%$ & $0.67 \%$ & $0.08 \%$ & $0.08 \%$ \\
\hline
\end{tabular}


Citation: Dahlbeck S, Hansen CC, deRiese W, Kagan AR, Torres C, et al. (2015) A Prospective Pilot Study of Single 19 Gy Fraction High-DoseRate Brachytherapy for Favorable-Risk Adenocarcinoma of the Prostate. J Nucl Med Radiat Ther 6: 215. doi: $10.4172 / 2155-9619.1000215$

Page 4 of 7

\begin{tabular}{|l|l|l|l|l|l|l|l|}
\hline \multicolumn{2}{|l|}{ Rectum } & $0.93 \%$ & $0.20 \%$ & $0 \%$ & $0.29 \%$ & $1.00 \%$ & $0.76 \%$ \\
\hline V75 & \multicolumn{7}{|l|}{} \\
\hline Prostate & $92.22 \%$ & $91.07 \%$ & $91.83 \%$ & $92.02 \%$ & $89.14 \%$ & $90.93 \%$ \\
\hline V100 & $51.45 \%$ & $45.30 \%$ & $52.47 \%$ & $60.87 \%$ & $55.20 \%$ & $57.46 \%$ \\
\hline V130 & $24.70 \%$ & $22.45 \%$ & $23.94 \%$ & $33.75 \%$ & $36.34 \%$ & $30.38 \%$ \\
\hline V160 & Abbreviations: V100 Percentage of the structure volume receiving 100\% of the prescription dose (19 Gy). & & \\
\hline
\end{tabular}

Table 3: Patient dose-volume-histogram constraints.

\section{Patient follow-up}

A phone call was placed the day after brachytherapy to check on the patient's status. Follow-up visits per protocol were done at 1 week and then scheduled every 3 months for the first 2 years, followed by every 6 months for the next 3 years. Each follow-up visit consisted of a history and physical examination, including a serum PSA (digital rectal exam performed if the PSA was elevated), plus completion of the prebrachytherapy questionnaires for comparison, and assessments for genitourinary (GU) and gastrointestinal (GI) toxicities were done using the National Cancer Institute's Common Terminology Criteria for Adverse Events (CTCAE) version 4.0. Biochemical relapse was defined according to recognized consensus guidelines, specifically, the Phoenix definition: PSA nadir plus $2 \mathrm{ng} / \mathrm{ml}$ [10]. No patients have been lost to follow-up.

\section{Statistical analysis}

The primary endpoints of this report were acute toxicity. Secondary endpoints were to assess quality of life, genitourinary, gastrointestinal, and sexual function as well as the treatment efficacy in terms of disease free survival (DFS), overall survival (OS), and biochemical control. Health-related quality of life and toxicity are reported using summary descriptive statistics and actuarial rates of sexual potency.

\section{Results}

A total of 6 patients met inclusion criteria and were enrolled in this pilot study between December 2012 and August 2013. Please see Table 4 for patient characteristics. The median age was 76 years old. The most common T stage was T1c, and the most common Gleason score was 7 . The pre-brachytherapy PSA mean was 7.8 , median 8.5 , and range 4.4-10.5. Only one patient received androgen deprivation therapy prior to radiotherapy. All patients received 19 Gy in a single fraction. Every patient was discharged home before $4 \mathrm{PM}$ on the same day of brachytherapy. Median follow-up for the group was 9 months (range, 6-12).

\begin{tabular}{|l|l|l|l|l|l|}
\hline Patient & Age & T stage & $\begin{array}{l}\text { Gleason } \\
\text { Pre-Tx }\end{array}$ & $\begin{array}{l}\text { PSA Pre- } \\
\text { Tx }\end{array}$ & ADT \\
\hline 1 & 70 & $1 \mathrm{c}$ & $3+3=6$ & 4.4 & No \\
\hline 2 & 76 & $2 \mathrm{a}$ & $4+3=7$ & 7.2 & No \\
\hline 3 & 77 & $1 \mathrm{c}$ & $3+3=6$ & 10.2 & No \\
\hline 4 & 76 & 2b & $3+4=7$ & 4.5 & No \\
\hline
\end{tabular}

\begin{tabular}{|l|l|l|l|l|l|}
\hline 5 & 81 & $1 \mathrm{c}$ & $4+3=7$ & 9.8 & No \\
\hline 6 & 74 & $2 \mathrm{c}$ & $3+4=7$ & 10.5 & Yes \\
\hline \multicolumn{7}{|l}{ Abbreviations: PSA : Prostate Specific Antigen; Pre-Tx : Pre-treatment; ADT : } \\
Androgen Deprivation Therapy prior to HDR brachytherapy \\
\hline
\end{tabular}

Table 4: Patient characteristics.

\section{Acute toxicity}

Data for acute toxicity is based on a median follow-up of 9 months for the 6 patients under evaluation. Genitourinary and gastrointestinal toxicities are reported in Figure 1. There were minimal low-grade toxicities observed, of which none persisted to the next follow-up appointment. No grade 3,4 or 5 toxicities were observed.
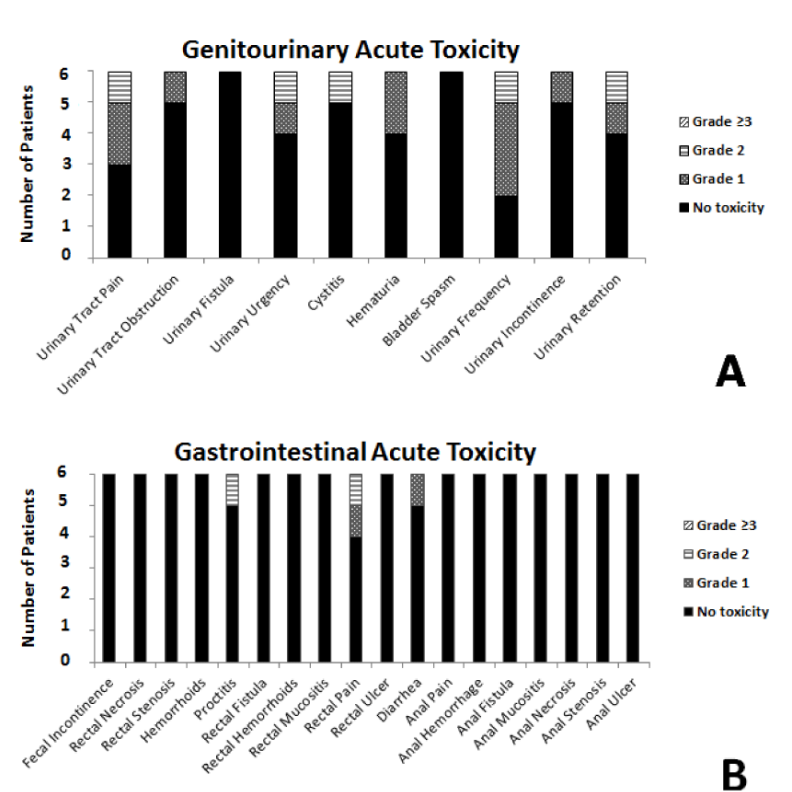

Figure 1: There were a total of 6 patients enrolled in this prospective pilot study GU. A) and GI. B) acute toxicities are reported with a median follow-up of 9 months.

Two of the 6 patients (33\%) experienced grade 2 genitourinary toxicity: one of the patients had grade 2 urinary frequency at the 6- 
Citation: Dahlbeck S, Hansen CC, deRiese W, Kagan AR, Torres C, et al. (2015) A Prospective Pilot Study of Single 19 Gy Fraction High-DoseRate Brachytherapy for Favorable-Risk Adenocarcinoma of the Prostate. J Nucl Med Radiat Ther 6: 215. doi: $10.4172 / 2155-9619.1000215$

Page 5 of 7

month follow-up appointment, which had resolved at the 9-month follow-up appointment, and the other patient had grade $2 \mathrm{GU}$ toxicities consisting of urinary tract pain, cystitis, and urinary urgency at the 1 week check-up and urinary retention at the 6-month check-up which all resolved at the next follow-up appointment. The second patient also experienced grade 2 GI toxicities in the form of proctitis and rectal pain at his 1-week check-up both of which had resolved at his 3-month follow-up.

\section{Health-related quality of life and care}

Please see Table 5 for quality of life data. Prior to treatment and at every follow-up visit, all 6 patients (100\%) had completed the questionnaires concerning bowel, urinary, and sexual quality of life. Overall perceived quality of life and quality of care questionnaires were also completed by all patients. Bowel complaints were highest at 3 months with the average score dropping to 91.9 from a baseline of 96.7 on the EPIC questionnaire; however, bowel quality of life did trend toward baseline at the 6-month follow-up and returned to baseline at the 9-month follow-up. Urinary complaints, as determined by the IPSS, reached a zenith within 3 months with an average 3.2 points above the baseline IPSS and then partially recovered by the 6-month follow-up.

\section{Sexual function assessment}

The International Index of Erectile Function (IIEF-5) questionnaire given before HDR brachytherapy revealed that all 6 patients had significant erectile dysfunction at baseline. Five of the six patients (83\%) reported baseline IIEF- 5 scores of $\leq 4$, where a score of $\leq 21$ is suggestive of erectile dysfunction. The other patient had a baseline IIEF-5 score of 10, yet was potent before HDR brachytherapy despite some dysfunction and disinterest. Sexual potency was preserved in the one patient who was potent preoperatively. Potency was defined as being able to achieve an erection sufficient for intercourse.

\section{Short-term biochemical control}

Biochemical relapse was defined as a PSA nadir plus $2 \mathrm{ng} / \mathrm{mL}$ [10]. The mean baseline PSA was $7.8 \mathrm{ng} / \mathrm{mL}$ (range, 4.4-10.5). Mean PSA levels gradually decreased from 7.8 at baseline to 1.4 at 9 months, and there have been no cases of biochemical failure (Table 5 ).

\begin{tabular}{|l|l|l|l|l|l|}
\hline Mean or Change in Mean Score (range) & Baseline & $\mathbf{1}$ week & $\mathbf{3}$ months & $\mathbf{6}$ months & $\mathbf{9}$ months \\
\hline Domain & $96.7(92.9-100)$ & $97.6(92.9-100)$ & $91.9(73.2-100)$ & $93.3(82.1-100)$ & $96.4(92.9-98.2)$ \\
\hline Bowel (EPIC) & $6.3(1$ to 12$)$ & $+4.2(-1$ to +15$)$ & $+3.2(-2$ to +14$)$ & $+2.5(0$ to +4$)$ & $+2(0$ to +4$)$ \\
\hline Urinary (IPSS) & $1.8(0$ to 5$)$ & $+0.8(-2$ to +4$)$ & $+0.3(0$ to +1$)$ & $-0.5(-1$ to 0$)$ & $0(-1$ to +1$)$ \\
\hline Bother (IPSS) & $74(60.5$ to 91$)$ & $+7.8(-5$ to +29.5$)$ & $+2.8(-11$ to +21.5$)$ & $-2.9(-11.5$ to +11$)$ & $+3.3(-10.5$ to +21.5$)$ \\
\hline Perceived Quality of Life & $4.3(1-5)$ & 5 & $4.8(4-5)$ & 5 & 5 \\
\hline Perceived Quality of Care & $7.8(4.4-10.5)$ & N/A & $1.9(0.6-4.8)$ & $1.1(0.5-1.9)$ & $1.4(0.4-2.7)$ \\
\hline Prostate Specific Antigen & Abbreviations: EPIC : Expanded Prostate Cancer Index Composite-Bowel Assessment; IPSS : International Prostate Symptom Score \\
\hline
\end{tabular}

Table 5: Health-related quality of life scores and biochemical control.

\section{Discussion}

The major findings of this report can be summarized as follows. First, we demonstrated the technical feasibility of a single-19 Gy fraction of HDR brachytherapy used as monotherapy in favorable-risk adenocarcinoma of the prostate. This is the first reported pilot study to investigate this single-fraction method in the United States, and the first-ever to do so without transperineal hyaluronic acid injections into the perirectal fat to displace the rectum. Second, our preliminary results suggest there is minimal to no acute low-grade GU or GI toxicity with this treatment and that quality of life measures are comparable to other common treatment modalities [11-14]. Furthermore, there were no acute high-grade GU or GI toxicities (Grade $\geq 3$ ). Third, biochemical control rates have been encouraging with good biochemical response to therapy and no biochemical relapses at a median follow-up of 9 months; however, it is far too early to report on this method's durable biochemical control rate. Finally, the cost of this treatment is substantially less than current alternative options. The actual cost for this single fraction therapy, reported by our institution's Southwest Cancer Center, is $\$ 15,096.00$. Likewise, a typical prostate cancer intensity-modulated radiation therapy treatment reportedly costs Medicare $\$ 31,574$ [15]. While the drastic difference in cost is easily appreciated, similar ranges exist when comparing any brachytherapy treatment to IMRT. Reason would follow that a single fraction of HDR brachytherapy would cost less than multiple fractions over a period of days. The economics of singlefraction HDR brachytherapy coupled with the convenience to the patient makes this an attractive treatment option, especially if durable control rates prove to be non-inferior to the current alternatives.

Prada et al. were the first to report on this HDR brachytherapy method using a single-19 Gy fraction as monotherapy for prostate cancer [9]. At a median follow-up of 9 months, their report had no Grade 2 or greater acute GI or GU toxicity and no chronic toxicities were observed. Chronic toxicity was defined as symptoms persisting or appearing beyond 6 months. Similar to Prada's results, we also found that this treatment had minimal acute GI or GU toxicities wherein we did not observe any Grade 3 or above adverse sequelae. One major difference between our pilot study and the trial performed by Prada was that we did not use the transperineal hyaluronic acid injections into the perirectal fat to displace the rectum. It was felt that this technique was unnecessary to minimize the dose to the rectum and prevent GI toxicity. In contrast to Prada's study, however, we did observe one patient (16.7\%) with temporary acute Grade 2 proctitis and rectal pain. Whether or not the hyaluronic acid injections would 
have prevented this single case of temporary acute GI toxicity is difficult to determine but is unlikely to warrant transperineal hyaluronic acid injections.

Actuarial biochemical control rates in Prada's study were 100\% and $88 \%$, respectively, for low- and intermediate-risk prostate cancer groups at 32 months. Although it is far too early to conclude anything concerning the durable biochemical control rates in the present study, there has been $100 \%$ biochemical control at a median follow-up of 9 months.

There is a paucity of literature concerning health-related quality of life (HRQOL) data for HDR monotherapy. There are, however, a few prospective multicenter cohort studies that have utilized validated HRQOL questionnaires to measure quality of life issues after LDR brachytherapy, external beam radiotherapy, and prostatectomy [11-14]. It appears that all of these treatment modalities commonly affect three major HRQOL domains - sexual, urinary, and bowel - in similar fashion. The greatest impact of HRQOL in these previously cited studies occurs within the first 6 months. There is typically an initial decline in quality of life within the first 3 months and then there is an appreciable recovery which typically plateaus after 1 year. Barkati et al. an Australian group studying HDR monotherapy, used the patient-reported modified EPIC questionnaire [16]. They showed that urinary and bowel domains were affected similarly with the majority of symptoms reported within the first 3 months after treatment. Similarly, we observed the most significant decrease in urinary and bowel quality of life at 3 months after treatment and then appreciated a return towards baseline thereafter. While it is known that radiation complications have variable responses over time, it is important to remember that an absence of complications does not rule out late toxicity. Clinicians have demonstrated that late complications can arise even though the patient was asymptomatic weeks and months earlier [17]. Of interest, the sexual domain was the most commonly affected in Barkati's study and the impact persisted beyond 1 year after treatment. Of our 6 patients, only one was sexually potent before treatment and has remained so 6 months after brachytherapy with no change in his IIEF-5 score of 10 .

Ghilezan et al. treated favorable-risk prostate cancer with HDR monotherapy to 24-27 Gy delivered in two fractions within 1 day. Their results were encouraging given the minimal acute ( $<6$ months) and chronic ( $>6$ months) toxicities and the fact that, like our study, the entire treatment was delivered in 1 day. Similar to their study, urinary frequency and urgency were the most common acute GU toxicities our patients experienced. Although acute GI toxicities were essentially non-existent in the Ghilezan report, they did appreciate a few chronic GI toxicities, however, the percentage of patients experiencing grade 2 or above was only $1 \%$ [18].

There are two major advantages of this treatment that we would like to highlight. First, it is arguably the most convenient and most time conserving treatment option for the patient. All 6 of our patients tolerated the procedure with minimal discomfort and were able to return home by $4 \mathrm{PM}$ the same day. The second major advantage is that it is arguably the least expensive treatment option available and deals with the economics of prostate cancer. Currently, in the United States, a major goal of health reform is to reduce the growth rate of healthcare expenses while simultaneously maintaining or improving the quality of care [18]. Prostate cancer treatment is an important area for comparative effectiveness research because its cost to healthcare in the United States is an estimated $\$ 4.5$ billion for the initial treatment alone [19]. To drive home this issue's relevance, the Institute of
Medicine's Committee on Comparative Effectiveness Research recently identified the treatment of favorable-risk prostate cancer as a top research priority [20]. Thus, it would be prudent to develop effective and affordable treatments, such as the present treatment method, to provide risk-adverse patients with a low-cost alternative to active surveillance.

This study does have limitations, the first of which being its limited follow-up period (median 9 months). As data matures, we will report the outcomes of chronic toxicity, disease free survival, overall survival, and biochemical control rates. However, given the pressing need for affordable and effective treatments in a cost-conscious healthcare system, we felt the study would add positively to the prostate cancer community discussion and be hypothesis generating.

We conclude that HDR brachytherapy delivered in a single-19 Gy fraction and used as monotherapy for favorable-risk prostate cancer is technically feasible with excellent patient satisfaction. No grade 3, 4 or 5 acute GI or GU toxicities were observed. Biochemical control appears to be comparable to other radiotherapy treatments (although the data is too immature to comment on durable biochemical control rates). The cost and convenience advantages of this treatment method make it an attractive option although our findings need to be confirmed in a larger prospective trial.

\section{References}

1. Kupelian PA, Potters L, Khuntia D, Ciezki JP, Reddy CA, et al. (2004) Radical prostatectomy, external beam radiotherapy $<72 \mathrm{~Gy}$, external beam radiotherapy $>$ or $=72 \mathrm{~Gy}$, permanent seed implantation, or combined seeds/external beam radiotherapy for stage T1-T2 prostate cancer. Int J Radiat Oncol Biol Phys 58: 25-33.

2. Yamada Y, Rogers L, Demanes DJ, Morton G, Prestidge BR, et al. (2012) American Brachytherapy Society consensus guidelines for high-dose-rate prostate brachytherapy. Brachytherapy 11: 20-32.

3. Demanes DJ, Martinez AA, Ghilezan M, Hill DR, Schour L, et al. (2011) High-dose-rate monotherapy: safe and effective brachytherapy for patients with localized prostate cancer. Int J Radiat Oncol Biol Phys 81: 1286-1292.

4. Mohammed N, Kestin L, Ghilezan M, Krauss D, Vicini F, et al. (2012) Comparison of acute and late toxicities for three modern high-dose radiation treatment techniques for localized prostate cancer. Int J Radiat Oncol Biol Phys 82: 204-212.

5. Grills IS, Martinez AA, Hollander M, Huang R, Goldman K, et al. (2004) High dose rate brachytherapy as prostate cancer monotherapy reduces toxicity compared to low dose rate palladium seeds. J Urol 171: 1098-1104.

6. Duchesne GM, Peters LJ (1999) What is the alpha/beta ratio for prostate cancer? Rationale for hypofractionated high-dose-rate brachytherapy. Int J Radiat Oncol Biol Phys 44: 747-748.

7. Williams SG, Taylor JM, Liu N, Tra Y, Duchesne GM, et al. (2007) Use of individual fraction size data from 3756 patients to directly determine the alpha/beta ratio of prostate cancer. Int J Radiat Oncol Biol Phys 68: 24-33.

8. King CR, Fowler JF (2001) A simple analytic derivation suggests that prostate cancer alpha/beta ratio is low. Int J Radiat Oncol Biol Phys 51: 213-214.

9. Prada PJ, Jimenez I, Gonzalez-Suarez H, Fernández J, Cuervo-Arango C (2012) High-dose-rate interstitial brachytherapy as monotherapy in one fraction and transperineal hyaluronic acid injection into the perirectal fat for the treatment of favorable stage prostate cancer: treatment description and preliminary results. Brachytherapy 11: 105-110.

10. Roach M 3rd, Hanks G, Thames H Jr., Schellhammer P, Shipley WU, et al. (2006) Defining biochemical failure following radiotherapy with or without hormonal therapy in men with clinically localized prostate 
Citation: Dahlbeck S, Hansen CC, deRiese W, Kagan AR, Torres C, et al. (2015) A Prospective Pilot Study of Single 19 Gy Fraction High-DoseRate Brachytherapy for Favorable-Risk Adenocarcinoma of the Prostate. J Nucl Med Radiat Ther 6: 215. doi: 10.4172/2155-9619.1000215

Page 7 of 7

cancer: recommendations of the RTOG-ASTRO Phoenix Consensus Conference. Int J Radiat Oncol Biol Phys. 65: 965-974.

11. Downs TM, Sadetsky N, Pasta DJ, Grossfeld GD, Kane CJ, et al. (2003) Health related quality of life patterns in patients treated with interstitial prostate brachytherapy for localized prostate cancer--data from CaPSURE. J Urol 170: 1822-1827.

12. Potosky AL, Davis WW, Hoffman RM, Stanford JL, Stephenson RA, et al. (2004) Five-year outcomes after prostatectomy or radiotherapy for prostate cancer: the prostate cancer outcomes study. J Natl Cancer Inst 96: 1358-1367.

13. Madalinska JB, Essink-Bot ML, de Koning HJ (2001) Health-related quality-of-life effects of radical prostatectomy and primary radiotherapy for screen-detected or clinically diagnosed localized prostate cancer. J Clin Oncol 19: 1619-1628.

14. Hoppe BS, Michalski JM, Mendenhall NP, Morris CG, Henderson RH, (2013) Comparative effectiveness study of patient-reported outcomes after proton therapy or intensity-modulated radiotherapy for prostate cancer. Cancer 120: 1076-1082.

15. Barkati M, Williams SG, Foroudi F, Tai KH, Chander S, et al. (2012) High-dose-rate brachytherapy as a monotherapy for favorable-risk prostate cancer: a Phase II trial. Int J Radiat Oncol Biol Phys 82: 1889-1896.

16. Ghilezan M, Martinez A, Gustason G, Krauss D, Antonucci JV, et al. (2012) High-dose-rate brachytherapy as monotherapy delivered in two fractions within one day for favorable/intermediate-risk prostate cancer: preliminary toxicity data. Int J Radiat Oncol Biol Phys 83: 927-932.

17. Perlroth DJ, Bhattacharya J, Goldman DP, Garber AM (2012) An economic analysis of conservative management versus active treatment for men with localized prostate cancer. J Natl Cancer Inst Monogr 2012: 250-257.

18. Mariotto AB, Yabroff KR, Shao Y, Feuer EJ, Brown ML (2011) Projections of the cost of cancer care in the United States: 2010-2020. J Natl Cancer Inst 103: 117-128.

19. Nguyen PL, Gu X, Lipsitz SR, Choueiri TK, Choi WW, et al. (2011) Cost implications of the rapid adoption of newer technologies for treating prostate cancer. J Clin Oncol 29: 1517-1524.

20. Heemsbergen WD, Peeters ST, Koper PC, Hoogeman MS, Lebesque JV (2006) Acute and late gastrointestinal toxicity after radiotherapy in prostate cancer patients: consequential late damage. Int J Radiat Oncol Biol Phys 66: 3-10. 